\title{
An Efficient Online Estimation Algorithm with Measurement Noise for Time-Varying Quantum States
}

\author{
Kun Zhang ${ }^{\mathrm{a}}$, Shuang Cong ${ }^{\mathrm{a}}$, Kezhi Li ${ }^{\mathrm{b}}$ \\ ${ }^{a}$ Department of Automation, University of Science and Technology of China, Hefei 230027, China. \\ ${ }^{b}$ University College London, NW1 2DA, United Kingdom.
}

\begin{abstract}
Inspired by the online alternating direction multiplier method (OADM), we propose an efficient online quantum state estimation (QSE) algorithm (QSE-OADM) for recovering time-varying quantum states in this paper. Specifically, in QSE-OADM, the density matrix recovery subproblem and measurement noise minimization subproblem are divided and solved separately without running the algorithm iteratively, which makes the proposed method much more efficient than all previous works. In the numerical experiments, for a 4-qubit system, the proposed algorithm can achieve more than $97.57 \%$ (fidelity) estimation accuracy after 71 samples, and the average runtime of per estimation is $(4.19 \pm 0.41) \times 10^{-4}$ seconds, which reveals its superior performance comparing with existing online processing algorithms.
\end{abstract}

Keywords: online quantum state estimation; minimization multi-problem; optimization algorithm; online alternating direction multiplier method.

\section{Introduction}

Quantum state estimation (QSE), also known as quantum state tomography, is a fundamental problem in quantum state preparation, quantum computation and quantum state feedback control [1, 2, 3]. The aim of QSE is to reconstruct the quantum state $\rho$ from a number of measurement times [4]. The state of an $n$-qubit system usually can be described by a $d \times d\left(d=2^{n}\right)$ density matrix, which has the physical constraints of positive semidefinite and unit-trace Hermitian. QSE can be converted to an optimization problem with the constraints and solved by numerical methods [5, 6]. The commonly used methods of QSE to measure the quantum state are based on strong, destructive measurements [7]. As a consequence, people have to prepare a large number of identical copies of the original quantum state. Moreover, the measurement apparatus need to be reconfigured at each measurement. For real-time applications, however, both measuring process and computation need to be carried out in a very short time. Thus such strong measurement is not deemed suitable for online QSE [8].

Continuous weak measurement (CWM) provides a new approach to estimate quantum states continuously [7, 9]. In the measurement process, by using CWM it is possible to gain the measurement information regarding the estimated state without disturbing it substantially, and the quantum state can be recovered by computing the ensemble averaging [10]. Due to the non-complete destructive characteristic of CWM, the online estimation of time-varying quantum states becomes feasible.

Email address: email: scong@ustc.edu.cn (Shuang Cong) 
Many optimization algorithms have been developed for offline QSE. For offline estimation of a fixed quantum state, there are a series of optimization algorithms based on the alternating direction multiplier method (ADMM) [11], which is an offline computing framework that effectively solves the optimization problem with separable objective functions. Li et al. adopted ADMM to solving QSE by ignoring the constraints of quantum density matrix [12]. Considering partial quantum state constraints, Zheng et al. and Zhang et al. proposed FP-ADMM based on fixed point equation [13] and IST-ADMM based on iterative shrinkage-thresholding [14], respectively. After that, Zhang et al. proposed three improved ADMM optimization algorithms for different types of interference in the quantum system [15, 16, 17]. All the above offline QSE algorithms need to pass the same set of measurement data through multiple iterations to estimate a fixed quantum state.

For online QSE, its estimated state is a more general state of dynamic evolution. In contrast, people usually used picture transformation to simplify the online problem of estimating dynamic states to a static problem of estimating the initial state [7, 10, 18]. Throughout the online QSE process in their researches, the estimation state is always the initial state, and then the dynamic state was obtained by the evolutionary model. Yang et al. designed an online estimation algorithm for dynamic quantum states [19]. They converted the state estimation problem at each sampling time into a constrained least square problem and solved it using the convex optimization toolbox (CVX) in MATLAB [20]. Since CVX is an offline optimization tool, the algorithm proposed by Yang (called CVX-LS) was essentially a double-loop algorithm, that is, changing the optimization problem online in the outer loop, and performing offline iterative optimization in the inner loop, which is very time-consuming. In terms of the online algorithm which focuses on processing data in sequence or incremental way, Youssry et al. proposed an online quantum state learning algorithm based on matrix exponential gradient method (MEG) [21]. MEG guarantees the positive semidefiniteness of the estimated matrix by adding the gradient update rule of logarithmic and exponential operations. More recently, Zhang et al. proposed an online QSE algorithm combined with the online proximal gradientADMM (OPG-ADMM) framework [22]. OPG-ADMM performs an online proximal gradient update by adding a proximal term about the density matrix. Essentially, both MEG and OPG-ADMM resort first-order stochastic gradient information to estimate the quantum state density matrix online. The main difference is that OPG-ADMM adopts an adaptive learning rate and exploits the ADMM framework, while the learning rate of MEG is a constant.

Considering the real-time application of QSE (such as quantum state feedback control), the currently sampled quantum state is expected to be reconstructed instantly. It motivates us to develop a real online QSE algorithm, which is able to efficiently complete dynamic state reconstructions at each sampling time. In other words, the goal of the online QSE algorithm is to track the time-varying quantum state in real time. The task of real-time reconstruction for the dynamic state is challenging. Because in each weak measurement sampling, only one noisy measurement value can be obtained. Meanwhile, the online algorithm only performs one iteration at each estimation, and the estimated density matrix has to meet the quantum state constraints.

In this paper, we propose a novel online estimation algorithm to achieve an accurate reconstruction of dynamic quantum state from noisy measurements. The online QSE problem is divided into two subproblems that can be solved by online alternating direction multiplier method (OADM) [23] which is an online variation framework of ADMM. One subproblem is the minimization of a quadratic problem subject to quantum state constraints for estimating the dynamic $\rho$, and the other is the minimization of an unconstrained problem for estimating the measurement noise $e$. Since the involved quantum state constraints make the direct solution of the subproblem of $\rho$ cumbersome, we simplify the solution by two steps: first solving an unconstrained subproblem, then updating the solution subject to the constraints through projection. The proposed QSE-OADM algorithm processes the measurement values in a serialized manner, and exactly solves two 
sub-problems in every estimation. Our proposed algorithm is used to estimate the dynamic state density matrix of multi-qubit systems, and compares its performance with existing algorithms for online QSE.

The rest of this paper is organized as follows. Section. 2 introduces the stochastic open quantum system evolution model in CWM. Section. 3 describes the optimization problem of online QSE with measurement noise. The QSE-OADM algorithm is proposed in Section. 4 Numerical experiments are carried out in Section. 5. Finally, a conclusion is drawn in Section. 6

\section{Stochastic open quantum system model in CWM}

\subsection{Discrete evolution model of $n$-qubit system}

The open quantum system can be described by the continuous stochastic master equation in Schrödinger picture as [10]:

$$
\begin{aligned}
& \rho(t+\Delta t)-\rho(t)=-\frac{i}{\hbar}[H, \rho(t)] \Delta t \\
& +\sum\left[L \rho(t) L^{\dagger}-\frac{1}{2}\left(L^{\dagger} L \rho(t)+\rho(t) L^{\dagger} L\right)\right] \Delta t \\
& +\sqrt{\eta} \sum\left[L \rho(t)+\rho(t) L^{\dagger}\right] d W,
\end{aligned}
$$

where $\rho(t) \in \mathbb{C}^{d \times d}$ denotes the quantum state density matrix; $H \in \mathbb{C}^{d \times d}$ is the Hamiltonian representing the total energy of the system; $L$ is a bounded operator pertaining to the Lindblad interaction; $L^{\dagger}$ denotes the conjugate transposition of $L ; \Delta t$ is the weak measurement time; $\hbar$ is set to $1 ; \eta$ is the measure efficiency; $d W$ denotes the noise produced by measurement output for zero error measurement and satisfies $E(d W)=0$, $E\left[(d W)^{2}\right]=\Delta t$; the last two terms of (1) represent the diffusion and backaction introduced by the measurement process.

The dynamic discrete evolution model of the stochastic master equation (11) can be obtained as [24]:

$$
\rho_{k+1}=\sum_{i=1}^{2^{n}} A_{i}(\Delta t) \rho_{k} A_{i}(\Delta t)^{\dagger},
$$

where $k=1,2, \ldots, N$ represents the sampling times; $A_{i}(\Delta t) \in \mathbb{C}^{d \times d}\left(i=1 \ldots 2^{n}\right)$ are the system evolution operators and can be constructed as:

$$
\begin{gathered}
A_{1}(\Delta t)=\underbrace{a_{0}(\Delta t) \otimes \ldots \otimes a_{0}(\Delta t) \otimes a_{0}(\Delta t)}_{n}, \\
A_{2}(\Delta t)=\underbrace{a_{0}(\Delta t) \otimes \ldots \otimes a_{0}(\Delta t) \otimes a_{1}(\Delta t)}_{n}, \\
\vdots \\
A_{2^{n}}(\Delta t)=\underbrace{a_{1}(\Delta t) \otimes \ldots \otimes a_{1}(\Delta t) \otimes a_{1}(\Delta t)}_{n},
\end{gathered}
$$

in which $a_{0}(\Delta t):=I-\left(L_{1}^{\dagger} L_{1} / 2+i H_{1}\right) \Delta t+\sqrt{\eta} L_{1} \cdot d W$ and $a_{1}(\Delta t):=L_{1} \sqrt{\Delta t}+\sqrt{\eta} L_{1} \cdot d W$ are weak measurement operators derived through a two-level quantum system [25]; $H_{1}=H_{0}+u_{x} H_{x}, H_{0}$ is the free Hamiltonian, $H_{x}$ is the control Hamiltonian and $u_{x}$ is a external regulate constant value; $\otimes$ denotes Kronecker product; $L_{1}=\xi \sigma$, where $\xi$ is the system interaction strength, and $\sigma$ denotes the Pauli matrix which can be selected as one of $\left\{\sigma_{x}, \sigma_{y}, \sigma_{z}, I\right\}$, where $\sigma_{x}=\left(\begin{array}{cc}0 & 1 \\ 1 & 0\end{array}\right), \sigma_{y}=\left(\begin{array}{cc}0 & -i \\ i & 0\end{array}\right), \sigma_{z}=\left(\begin{array}{cc}1 & 0 \\ 0 & -1\end{array}\right)$, and $I$ is the $2 \times 2$ identity matrix. 
The corresponding discrete evolution of the measurement operators of an $n$-qubit system (1) can be described by:

$$
M_{k+1}=\sum_{i=1}^{2^{n}} B_{i}(\Delta t) M_{k} B_{i}(\Delta t)^{\dagger},
$$

where $B_{i}(\Delta t) \in \mathbb{C}^{d \times d}\left(i=1 \ldots 2^{n}\right)$ are the weak measurement operators and can be constructed as:

$$
\begin{gathered}
B_{1}(\Delta t)=\underbrace{b_{0}(\Delta t) \otimes \ldots \otimes b_{0}(\Delta t) \otimes b_{0}(\Delta t)}_{n}, \\
B_{2}(\Delta t)=\underbrace{b_{0}(\Delta t) \otimes \ldots \otimes b_{0}(\Delta t) \otimes b_{1}(\Delta t)}_{n}, \\
B_{2^{n}}(\Delta t)=\underbrace{b_{1}(\Delta t) \otimes \ldots \otimes b_{1}(\Delta t) \otimes b_{1}(\Delta t)}_{n},
\end{gathered}
$$

in which $b_{0}(\Delta t):=I-\left(L_{1}^{\dagger} L_{1} / 2+i H_{1}\right) \Delta t$ and $b_{1}(\Delta t):=L_{1} \sqrt{\Delta t}$ [25].

\subsection{Construction of the measurement record sequence}

The measurement record sequence consists of the measurement values obtained at all sampling times. For convenience, people usually construct the measurement values of CWM in Heisenberg picture [7, [10, [18]. At the current sampling time $k$, the measurement record sequence is the measurement operators $\left\{\tilde{M}_{i}\right\}_{i=1}^{k} \in \mathbb{C}^{d \times d}$ acting on the initial quantum state density matrix $\rho_{1}$ as:

$$
y_{i}=\operatorname{tr}\left(\tilde{M}_{i}^{\dagger} \rho_{1}\right), \quad i=1,2, \ldots, k,
$$

where $\operatorname{tr}(X)$ denotes the sum of the diagonal elements of the matrix $X$.

Note that the construction method of measurement operators in 6 is $\tilde{M}_{k+1}=\sum_{j=1}^{2^{n}} B_{j}(\Delta t)^{\dagger} \tilde{M}_{k} B_{j}(\Delta t)$, which is different from (4) proposed in this paper. Moreover, it can be observed from (6) that the measurement values $\left\{y_{i}\right\}_{i=1}^{k}$ at each sampling time are related to the initial quantum state density matrix $\rho_{1}$. Therefore, under the construction method of (6), the online estimation state is always the unchanged initial state $\rho_{1}$. While the estimated state $\hat{\rho}_{k}$ at the sampling time $k$ is obtained by the estimated initinal state evolving $k$ steps according to the system evolution model (2).

In Schrödinger picture, the measurement record sequence equivalent to (6) is the initial measurement operator $M_{1}$ acting on the quantum states $\left\{\rho_{i}\right\}_{i=1}^{k}$ as [26]:

$$
y_{i}=\operatorname{tr}\left(M_{1}^{\dagger} \rho_{i}\right), \quad i=1,2, \ldots, k,
$$

Here, we propose another method for constructing the measurement record sequence as:

$$
\begin{aligned}
y_{i} & =\operatorname{tr}\left(M_{k-i+1}^{\dagger} \rho_{k}\right)=\operatorname{vec}\left(M_{k-i+1}\right)^{\dagger} \operatorname{vec}\left(\rho_{k}\right), \\
i & =1, \ldots, k,
\end{aligned}
$$

where $\operatorname{vec}(X)$ represents the sequential combination of all the columns of the matrix $X$ into a column vector.

The proposed construction method (8) correlates the measurement values $\left\{y_{i}\right\}_{i=1}^{k}$ at each sampling time with the quantum state density matrix $\rho_{k}$, avoiding the need to evolve through the system evolution model after each estimation to obtain $\hat{\rho}_{k}$. This makes it possible for estimated density matrix to be the quantum state at the current sampling time. Table 1 shows the measurement values $\left\{y_{i}\right\}_{i=1}^{k}$ and the measurement record sequence $b_{k}$ at each sampling time. As one can see from Table $1 ; b_{k}$ is composed of $\left[y_{1}, \ldots, y_{i}, \ldots y_{k}\right]$, where $y_{1}=\operatorname{tr}\left(M_{k}^{\dagger} \rho_{k}\right), y_{2}=\operatorname{tr}\left(M_{k-1}^{\dagger} \rho_{k}\right), \ldots, y_{k}=\operatorname{tr}\left(M_{1}^{\dagger} \rho_{k}\right)$. Note that the measurement operator $\left\{M_{i}\right\}_{i=1}^{k}$ is arranged in reverse order from $M_{k}$ to $M_{1}$ relative to the quantum state $\rho_{k}$. 
Table 1: The Construction Process of Measurement Record Sequence

\begin{tabular}{llllll}
\hline & $y_{1}$ & $y_{2}$ & $y_{3}$ & $\ldots$ & $y_{k}$ \\
\hline$b_{1}$ & $\operatorname{tr}\left(M_{1}^{\dagger} \rho_{1}\right)$ & & & & \\
$b_{2}$ & $\operatorname{tr}\left(M_{2}^{\dagger} \rho_{2}\right)$ & $\operatorname{tr}\left(M_{1}^{\dagger} \rho_{2}\right)$ & & & \\
$b_{3}$ & $\operatorname{tr}\left(M_{3}^{\dagger} \rho_{3}\right)$ & $\operatorname{tr}\left(M_{2}^{\dagger} \rho_{3}\right)$ & $\operatorname{tr}\left(M_{1}^{\dagger} \rho_{3}\right)$ & & \\
$\vdots$ & $\vdots$ & $\vdots$ & $\vdots$ & & \\
$b_{k}$ & $\operatorname{tr}\left(M_{k}^{\dagger} \rho_{k}\right)$ & $\operatorname{tr}\left(M_{k-1}^{\dagger} \rho_{k}\right)$ & $\operatorname{tr}\left(M_{k-2}^{\dagger} \rho_{k}\right)$ & $\ldots$ & $\operatorname{tr}\left(M_{1}^{\dagger} \rho_{k}\right)$ \\
\hline
\end{tabular}

\section{Problem statement of online QSE}

In order to make full use of the measurement record sequence and alleviate the computational burden in the online processing, a sliding window containing the most recent measurements is adopted in consideration. Therefore, we rewrite the measurement record sequence as:

$$
b_{k}=\left(y_{\max (1, k-l+1)}, \ldots, y_{k-1}, y_{k}\right)^{\mathrm{T}},
$$

where $l$ is the size of the sliding window and $\max (1, k-l+1)$ represents the starting index of the window.

When the number of obtained measurements is less than $l$, the size of the window is equal to the number of sampling times $k$. Otherwise, the size of sliding window remains $l$ (containing the $l$ most recent measurements). The update strategy of the sliding window is First-In-First-Out (FIFO), which allows the online stream of measurements to be incorporated into the model, while gradually removing old measurements.

According to (8), we construct the sampling matrix corresponding to (9) as:

$$
\mathcal{A}_{k}=\left(\operatorname{vec}\left(M_{\min (k, l)}\right), \ldots, \operatorname{vec}\left(M_{2}\right), \operatorname{vec}\left(M_{1}\right)\right)^{\dagger} .
$$

It is worth noting that when the number of samplings is greater than or equal to $l$, the sampling matrix $\mathcal{A}_{k}$ remains unchanged. At the same time, considering the measurement noise in the actual weak measurement process, using the sampling matrix $\mathcal{A}_{k}$ and the density matrix $\rho_{k}$, we can rewrite $b_{k}$ as $b_{k}=\mathcal{A}_{k} \operatorname{vec}\left(\rho_{k}\right)+e_{k}$, where $e_{k} \in \mathbb{R}^{\min (k, l) \times 1}$ is the measurement noise and is assumed to be Gaussian noise.

We first define the quadratic pseudo-norm as $\|x\|_{P}^{2}=x^{\dagger} P x$, where $x \in \mathbb{C}^{m \times 1}$ is a vector of decision variables and $P \in \mathbb{C}^{m \times m}$ is any symmetric positive definite weight matrix. Therefore, at the sampling time $k$, the online QSE can be formulated as a convex optimization problem with constraints:

$$
\begin{array}{ll}
\min _{\hat{\rho}, \hat{e}} & \left\|\operatorname{vec}\left(\hat{\rho}-\hat{\rho}_{k-1}\right)\right\|_{w I_{1}}^{2}+I_{C}(\hat{\rho})+\|\hat{e}\|_{\gamma I_{2}}^{2}, \\
\text { s.t. } & \mathcal{A}_{k} \operatorname{vec}(\hat{\rho})+\hat{e}=b_{k},
\end{array}
$$

where $\hat{\rho}$ denotes the quantum state density matrix to be estimated; $\hat{e}$ denotes the measurement noise to be filtered; $\left\|\operatorname{vec}\left(\hat{\rho}-\hat{\rho}_{k-1}\right)\right\|_{w I_{1}}^{2}$ represents the distance between the estimated state $\hat{\rho}$ at current sampling time $k$ and the previous state estimation $\hat{\rho}_{k-1}$, which reflects that the online estimation doesn't want to forget what has been learned too far; $\|\hat{e}\|_{\gamma I_{2}}^{2}$ means to reduce the measurement error of the current estimated state, and it is also the common way of denoising; the weight matrices of the quadratic pseudo-norm are specifically taken as $w I_{1}$ and $\gamma I_{2}$, respectively, in which $w>0$ and $\gamma>0$ are weight parameters; $I_{1}$ and $I_{2}$ denote identity matrices with dimensions $d^{2}$ and $\min (k, l)$, respectively; convex set $C:=\left\{\hat{\rho} \geq 0, \operatorname{tr}(\hat{\rho})=1, \hat{\rho}^{\dagger}=\hat{\rho}\right\}$ represents the quantum state constraints; when the estimated state satisfies constraints $C$, the indicator function $I_{C}(\hat{\rho})$ equals 0 , otherwise $I_{C}(\hat{\rho})$ is $\infty$. 


\section{Online QSE Algorithm with measurement noise}

We introduce the online alternating direction multiplier method (OADM) [23] to develop the online QSE algorithm. For the constrained online convex optimization problem (11) with separable two-objective variables $\hat{\rho}$ and $\hat{e}$, the basic idea of OADM is to decompose it into two sub-problems and solve them alternately. The framework of OADM is to minimize the corresponding augmented Lagrangian functions of the two primal variables in turn, and finally update the Lagrangian multiplier by dual gradient ascent. Furthermore, only one update is required to calculate for the primal variables and the Lagrange multiplier after each sampling time $k$.

The augmented Lagrangian of (11) is $L_{k}(\hat{\rho}, \hat{e}, \lambda):=I_{C}(\hat{\rho})+\left\|\operatorname{vec}\left(\hat{\rho}-\hat{\rho}_{k-1}\right)\right\|_{w I_{1}}^{2}+\|\hat{e}\|_{\gamma I_{2}}^{2}-\left\langle\lambda, \mathcal{A}_{k} \operatorname{vec}(\hat{\rho})+\right.$ $\left.\hat{e}-b_{k}\right\rangle+(\alpha / 2)\left\|\mathcal{A}_{k} \operatorname{vec}(\hat{\rho})+\hat{e}-b_{k}\right\|_{2}^{2}$, where $\lambda$ is the Lagrange multiplier and $\alpha>0$ is the penalty parameter.

Thus, in the OADM for online QSE at the sampling time $k$, the estimated density matrix $\hat{\rho}_{k}$, Gaussian noise $\hat{e}_{k}$, and the Lagrange multiplier $\lambda_{k}$ are respectively:

$$
\left\{\begin{aligned}
\hat{\rho}_{k}= & \arg \min _{\hat{\rho}}\left\{\frac{\alpha}{2}\left\|\mathcal{A}_{k} \operatorname{vec}(\hat{\rho})+\hat{e}_{k-1}-b_{k}-\lambda_{k-1} / \alpha\right\|_{2}^{2}\right. \\
& \left.+I_{C}(\hat{\rho})+\left\|\operatorname{vec}\left(\hat{\rho}-\hat{\rho}_{k-1}\right)\right\|_{w I_{1}}^{2}\right\} \\
\hat{e}_{k}= & \arg \min _{\hat{e}}\left\{\|\hat{e}\|_{\gamma I_{2}}^{2}\right. \\
& \left.+\frac{\alpha}{2}\left\|\mathcal{A}_{k} \operatorname{vec}\left(\hat{\rho}_{k}\right)+\hat{e}-b_{k}-\lambda_{k-1} / \alpha\right\|_{2}^{2}\right\} \\
\lambda_{k}= & \lambda_{k-1}-\alpha\left(\mathcal{A}_{k} \operatorname{vec}\left(\hat{\rho}_{k}\right)+\hat{e}_{k}-b_{k}\right) .
\end{aligned}\right.
$$

Note that index $k$ represents the sampling times in which we apply the continuous measurement as well as the estimation updates. Therefore, only a single iteration is performed for (12) so as to devise a computationally minimalistic method suitable for real-time implementation.

In the following, we explicitly provide efficient methods for solving the two optimization problems by updating primal variables $\hat{\rho}, \hat{e}$ in (12a), (12b). The Lagrangian multiplier $\lambda$ can be directly updated by (12c).

Update $\hat{\rho}_{k}$ : For the subproblem of quantum state density matrix, it contains a non-differentiable indicator function about the quantum state and two quadratic terms, which are a least square penalty term and a quadratic pseudo-norm term, respectively.

Since the indicator function can be solved by projection [27]. Therefore, the solution process of $\hat{\rho}$ can be divided into two steps. In brief, we first solve a relatively simple unconstrained problem without considering the indicator function, and then obtain the estimated state $\hat{\rho}_{k}$ that satisfies the constraints of the quantum state by solving a projection problem.

Step 1: When neglecting the indicator function $I_{C}(\hat{\rho})$ and letting $\mathbf{u}$ denote the constant $\left(b_{k}+\lambda_{k-1} / \alpha-\hat{e}_{k-1}\right)$, the subproblem (12a) can be written as an unconstrained convex problem:

$$
\tilde{\rho}_{k}=\arg \min _{\hat{\rho}}\left\{\frac{\alpha}{2}\left\|\mathcal{A}_{k} \operatorname{vec}(\hat{\rho})-\mathbf{u}\right\|_{2}^{2}+\left\|\operatorname{vec}\left(\hat{\rho}-\hat{\rho}_{k-1}\right)\right\|_{w I_{1}}^{2}\right\}
$$

where $\tilde{\rho}_{k}$ denotes the estimated state is not bounded by the quantum state constraints.

Because all terms in (13) are differentiable, the optimal solution $\tilde{\rho}_{k}$ can be solved directly by first-order optimality condition: $\alpha \mathcal{A}_{k}^{\dagger}\left(\mathcal{A}_{k} \operatorname{vec}\left(\tilde{\rho}_{k}\right)-\mathbf{u}\right)+2 w \operatorname{vec}\left(\tilde{\rho}-\hat{\rho}_{k-1}\right)=0$, and we can get the optimal solution as:

$$
\operatorname{vec}\left(\tilde{\rho}_{k}\right)=\left(W^{-1}+\mathcal{A}_{k}^{\dagger} V^{-1} \mathcal{A}_{k}\right)^{-1}\left(W^{-1} \operatorname{vec}\left(\hat{\rho}_{k-1}\right)+\mathcal{A}_{k}^{\dagger} V^{-1} \mathbf{u}\right),
$$

where $\left(W^{-1}+\mathcal{A}_{k}^{\dagger} V^{-1} \mathcal{A}_{k}\right)$ is a non-singular matrix; $W=\frac{1}{2 w} I_{1}>0, V=\frac{1}{\alpha} I_{3}>0\left(\mathcal{A}_{k}^{\dagger} \mathcal{A}_{k}\right.$ is essentially a positive semidefinite matrix), and $I_{3}$ denotes the identity matrix with dynamic dimensions $\min (k, l)$. 
Based on the Matrix Inversion Lemma [28]:

$$
\begin{aligned}
& \left(W^{-1}+\mathcal{A}_{k}^{\dagger} V^{-1} \mathcal{A}_{k}\right)^{-1}=W-W \mathcal{A}_{k}^{\dagger}\left(V+\mathcal{A}_{k} W \mathcal{A}_{k}^{\dagger}\right)^{-1} \mathcal{A}_{k} W, \\
& \left(W^{-1}+\mathcal{A}_{k}^{\dagger} V^{-1} \mathcal{A}_{k}\right)^{-1} \mathcal{A}_{k}^{\dagger} V^{-1}=W \mathcal{A}_{k}^{\dagger}\left(V+\mathcal{A}_{k} W \mathcal{A}_{k}^{\dagger}\right)^{-1},
\end{aligned}
$$

the optimal solution (14) can be rewritten as:

$$
\begin{aligned}
\operatorname{vec}\left(\tilde{\rho}_{k}\right)= & \operatorname{vec}\left(\hat{\rho}_{k-1}\right)+ \\
& \mathcal{A}_{k}^{\dagger}\left[\left(\frac{2 w}{\alpha} I_{3}+\mathcal{A}_{k} \mathcal{A}_{k}^{\dagger}\right)^{-1}\left(\mathbf{u}-\mathcal{A}_{k} \operatorname{vec}\left(\hat{\rho}_{k-1}\right)\right)\right] .
\end{aligned}
$$

Step 2: Considering the indicator function $I_{C}(\hat{\rho})$, the solution $\hat{\rho}_{k}$ that satisfies the quantum state constraints can be solved by the following convex optimization problem:

$$
\begin{aligned}
\hat{\rho}_{k}= & \arg \min _{\hat{\rho}}\left\|\operatorname{vec}\left(\hat{\rho}-\tilde{\rho}_{k}\right)\right\|_{I_{1}}^{2}, \\
& \text { s.t. } \quad \hat{\rho} \geq 0, \operatorname{tr}(\hat{\rho})=1, \hat{\rho}^{\dagger}=\hat{\rho} .
\end{aligned}
$$

Using $\left(\tilde{\rho}_{k}+\tilde{\rho}_{k}^{\dagger}\right) / 2$ with Hermitian characteristics to replace $\tilde{\rho}_{k}$ in (17) to satisfy the constraint $\hat{\rho}^{\dagger}=\hat{\rho}$. Since it is the calculation of the density matrix elements, (17) reduces to the density matrix projection problem [27]:

$$
\begin{gathered}
\hat{\rho}_{k}=\arg \min _{\hat{\rho}}\left\|\hat{\rho}-\left(\tilde{\rho}_{k}+\tilde{\rho}_{k}^{\dagger}\right) / 2\right\|_{F}^{2}, \\
\text { s.t. } \quad \hat{\rho} \geq 0, \operatorname{tr}(\hat{\rho})=1,
\end{gathered}
$$

where $\|\cdot\|_{F}$ is the Frobenius norm.

Essentially, the problem of (18) is a nonlinear semidefinite programming problem and usually solved by interior-point method. Instead, we use a direct calculation method to solve it by the singular value decomposition. $\left(\tilde{\rho}_{k}+\tilde{\rho}_{k}^{\dagger}\right) / 2$ can be diagonalized into $U \operatorname{diag}\left\{a_{i}\right\} U^{\dagger}$ by unitary similarity, where $\operatorname{diag}\left\{a_{1}, \ldots, a_{d}\right\}$ is a diagonal matrix whose singular values are arranged in nonincreasing order; $U \in \mathbb{C}^{d \times d}$ is a unitary matrix. The optimal solution of (18) can be written as:

$$
\hat{\rho}_{k}=U \operatorname{diag}\left\{\sigma_{i}\right\} U^{\dagger},
$$

where diag $\left\{\sigma_{1}, \ldots, \sigma_{d}\right\}$ is the singular value diagonal matrix of $\hat{\rho}_{k}$ and $\left\{\sigma_{i}\right\}_{i=1}^{d}$ can be solved from

$$
\begin{aligned}
& \min _{\left\{\sigma_{i}\right\}} \frac{1}{2} \sum_{i=1}^{d}\left(\sigma_{i}-a_{i}\right)^{2}, \\
& \text { s.t. } \sum_{i=1}^{d} \sigma_{i}=1, \sigma_{i} \geq 0 .
\end{aligned}
$$

The Lagrange function of 20 is:

$$
L\left\{\sigma_{i}, \kappa_{i}, \beta\right\}=\frac{1}{2} \sum_{i=1}^{d}\left(\sigma_{i}-a_{i}\right)^{2}-\kappa_{i} \sigma_{i}+\beta\left(\sum_{i=1}^{d} \sigma_{i}-1\right),
$$

where $\left\{\kappa_{i}\right\}_{i=1}^{d}$ and $\beta$ are Langrange multipliers. 
For the convex optimization problem [20], we can find the optimal $\sigma_{i}^{*}, \kappa_{i}^{*}$, and $\beta^{*}$ by solving its KarushKuhn-Tucker (KKT) conditions [29] as: $\sigma_{i}^{*} \geq 0, \sum_{i=1}^{d} \sigma_{i}^{*}=1, \kappa_{i}^{*} \geq 0, \kappa_{i}^{*} \sigma_{i}^{*}=0$, and $\sigma_{i}^{*}-a_{i}-\kappa_{i}^{*}+\beta^{*}=0$ $(i=1, \ldots, d)$. By eliminating variables $\kappa_{i}^{*}$, we can get:

$$
\left\{\begin{array}{l}
\sigma_{i}^{*} \geq 0 \\
\sigma_{i}^{*} \geq a_{i}-\beta^{*}, \\
\sigma_{i}^{*}\left(\sigma_{i}^{*}-\left(a_{i}-\beta^{*}\right)\right)=0 \\
\sum_{i=1}^{d} \sigma_{i}^{*}=1 .
\end{array}\right.
$$

From (22b), we can discuss the soltion of $\sigma_{i}^{*}$ in two cases: 1) if $\sigma_{i}^{*}>a_{i}-\beta^{*}$, according to (22c) we can get that $\sigma_{i}^{*}=0$, and at the same time, $a_{i}<\beta^{*}$ is established;2) if $\sigma_{i}^{*}=a_{i}-\beta^{*}$, we can get $\sigma_{i}^{*}=a_{i}-\beta^{*}$, and at this time combined with 22a), there is $a_{i} \geq \beta^{*}$ holds. Thus, we can directly obtain:

$$
\sigma_{i}^{*}=\max \left(a_{i}-\beta^{*}, 0\right),
$$

where the optimal Langrange multiplier $\beta^{*}$ can be calculated from $\sum_{i=1}^{d} \max \left\{a_{i}-\beta^{*}, 0\right\}=1$ using the condition 22d].

Specifically, we let $\beta^{*}=a_{i}(i=1, \ldots, d)$ determine the interval in which the optimal $\beta^{*}$ belongs. Assuming that $a_{q}-\beta^{*} \geq 0$ and $a_{q+1}-\beta^{*}<0$ are established in $\left[a_{q}, a_{q+1}\right]$, the optimal $\beta^{*}$ can be calculated by $\sum_{i=1}^{q}\left(a_{i}-\beta^{*}\right)=1$ as:

$$
\beta^{*}=\left(\sum_{i=1}^{q} a_{i}-1\right) / q
$$

Update $\hat{e}_{k}:(12 \mathrm{~b})$ is an unconstrained quadratic program that admits an analytical solution directly from first-order optimality condition of $\hat{e}$. Thus, $\hat{e}_{k}$ is updated as:

$$
\hat{e}_{k}=(\alpha /(2 \gamma+\alpha))\left(b_{k}+\lambda_{k-1} / \alpha-\mathcal{A}_{k} \operatorname{vec}\left(\hat{\rho}_{k}\right)\right) .
$$
1

The summary of the online QSE algorithm (QSE-OADM) proposed in this paper is shown in Algorithm

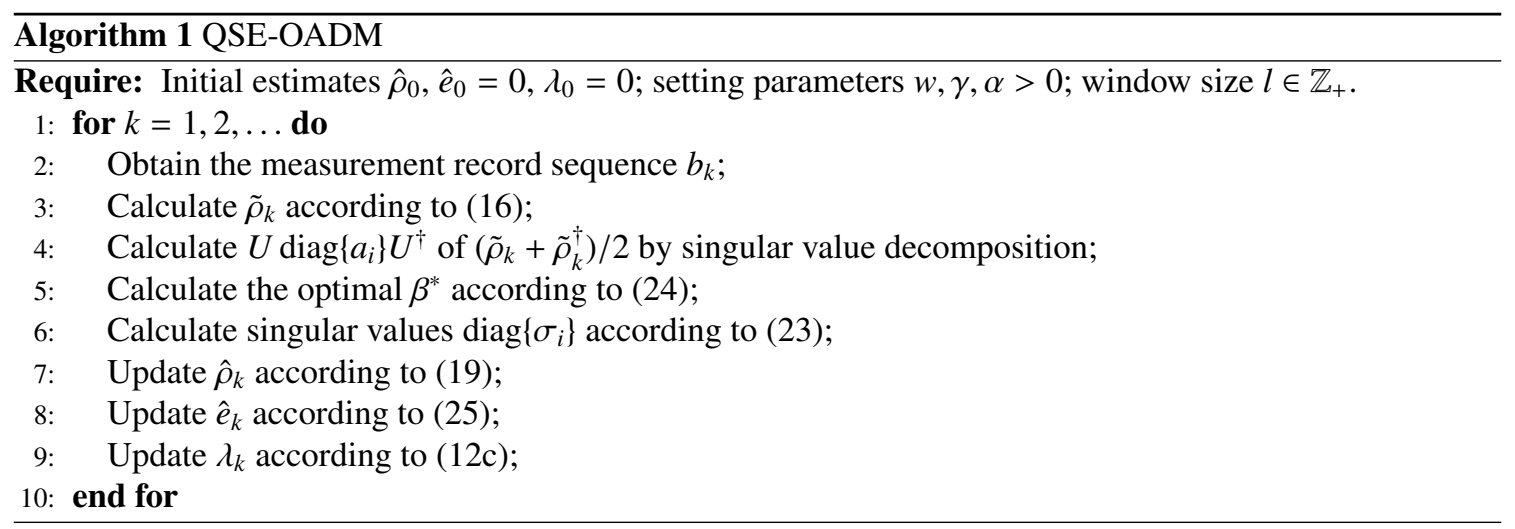

Compared with the offline QSE algorithm, whose estimation target is a fixed state, the estimation target $\rho_{k}$ of the online QSE algorithm based on CWM changes with time, which is a dynamic target estimation 
problem. Moreover, in some offline algorithms [12, 13, 14, 15, 16, 17], the estimated state is assumed to be sparse and low rank, while the quantum state estimated online is a more general state. In addition, the online algorithm obtains the estimated state $\hat{\rho}_{k}$ in only one iteration. The offline algorithm passes the same set of data through multiple iterations to finally get the state estimation.

Comparing the proposed QSE-OADM algorithm with CVX-LS [19], OPG-ADMM [22], and MEG [21] algorithms, the main difference is in the method of solving the density matrix subproblem. CVX-LS does not consider the measurement noise $e$ and uses the offline convex optimization toolbox as a solver, which is not practical for online processing. Both OPG-ADMM and MEG use the first-order information to approximately solve the subproblem of density matrix by exploiting the online proximal gradient descent method and the matrix exponential gradient method, respectively. Different from gradient-based approximation approaches, the algorithm QSE-OADM proposed in this paper decomposes the solution steps and exactly solves the estimated density matrix of the time-varying quantum state in every estimation, which makes the proposed method more efficient.

For the comparison of computational complexity, we compare the proposed QSE-OADM algorithm with OPG-ADMM and MEG in the overall online estimation. For the QSE-OADM algorithm, the update rule of the density matrix is $\left[16\right.$, where $\left(\frac{2 w}{\alpha} I_{3}+\mathcal{A}_{k} \mathcal{A}_{k}^{\dagger}\right) \operatorname{costs} O\left(l^{2} d^{2}\right)$ computational complexity (the computational complexity of its inversion is $O\left(l^{3}\right)$, which is smaller than $O\left(l^{2} d^{2}\right)$ because of $\left.l<d^{2}\right)$. Moreover, we recap that the sampling matrix $\mathcal{A}_{k}$ defined by (10) remains unchanged when the sampling times $k \geq l$, so $\left(\frac{2 w}{\alpha} I_{3}+\mathcal{A}_{k} \mathcal{A}_{k}^{\dagger}\right)$ only needs to be calculated $l$ times. Thus, the total computational complexity is $l * O\left(l^{2} d^{2}\right)$. The complexity of the singular value decomposition of the density matrix in $(19)$ is $O\left(d^{3}\right)$. Since the quantum state constraints are strictly guaranteed in every estimation, the total computational complexity is $N * O\left(d^{3}\right)$. In short, the overall computational complexity of QSE-OADM is $l * O\left(l^{2} d^{2}\right)+N * O\left(d^{3}\right)$. The overall computational complexity of OPG-ADMM is $l * O\left(d^{6}\right)+N * O\left(d^{3}\right)$, where $l * O\left(d^{6}\right)$ is the cost for the singular value decomposition of $\mathcal{A}_{k}^{\dagger} \mathcal{A}_{k}$ when calculating the adaptive learning rate; $N * O\left(d^{3}\right)$ is the computational complexity of solving the semidefinite programming problem with state constraints. The overall computational complexity of MEG is $2 N * O\left(d^{3}\right)$, which is the logarithmic operation and exponential operation of the density matrix (implemented by the singular value decomposition). Compared with the fixed sliding window size $l$, in actual online estimation, $N$ can be increased indefinitely, that is, $l \ll N$, so $l * O\left(l^{2} d^{2}\right)<l * O\left(d^{6}\right)<N * O\left(d^{3}\right)$. Therefore, the overall computational complexity of the QSE-OADM algorithm is less than that of the OPGADMM and MEG algorithms.

In fact, there are two types of interference in the quantum state, which are the noise in the measurement process and the sparse disturbance in the quantum state. We only study the online estimation algorithm with measurement noise in this paper. The study of both cases will be the future research work.

\section{Numerical experiments and results analysis}

In this section, numerical experiments are carried out to assess online properties of the proposed QSEOADM algorithm in the reconstruction performance of time-varying states. In the experiments, the measurement record sequence is constructed by $b_{k}=\mathcal{A}_{k} \operatorname{vec}\left(\rho_{k}\right)+e_{k}$. The true quantum state $\rho_{k}$ of the estimated system is generated by (2). The corresponding sampling matrix $\mathcal{A}_{k}$ is defined by (10). For the discrete evolution model the $n$-qubit quantum system, the parameters are set as: $L_{1}=\xi \sigma_{z}, H_{1}=\sigma_{z}+u_{x} \sigma_{x}, u_{x}=2, \eta=0.5, \xi=$ $0.7, d W=0.001$. Without loss of generality, the signal-to-noise ratio (SNR) of the Gaussian measurement noise $e_{k}$ is $40 d B$. For online QSE, the number of sampling times is set to $N=500$. The initial state density matrix of the true $n$-qubit system is chosen as $\rho_{1}^{n}=\underbrace{\rho_{1} \otimes \ldots \otimes \rho_{1}}_{n}, \rho_{1}=[0.5,(1-i) /(\sqrt{8}) ;(1+i) /(\sqrt{8}), 0.5]$, 
and the initial estimate is selected as $\hat{\rho}_{1}^{n}=\underbrace{\hat{\rho}_{1} \otimes \ldots \otimes \hat{\rho}_{1}}_{n}, \hat{\rho}_{1}=[0,0 ; 0,1]$. The initial measurement operator is $M_{1}^{n}=\underbrace{\sigma_{z} \otimes \ldots \otimes \sigma_{z}}_{n}$ (the superscript $n$ indicates the number of qubits).

As for the estimated quantum state, the accuracy performance is assessed by two typical criteria. The first one is the fidelity, which is defined as:

$$
\text { fidelity }(k)=\operatorname{tr}\left(\sqrt{\sqrt{\hat{\rho}_{k}} \rho_{k} \sqrt{\hat{\rho}_{k}}}\right),
$$

where $\hat{\rho}_{k}$ is the estimated density matrix and $\rho_{k}$ is the true density matrix. (The range of fidelity is [0,1]. The closer to 1 , the more similar the estimated state and the true state are.)

The second one is the normailized distance $D\left(\hat{\rho}_{k}, \rho_{k}\right)$, which is defined as:

$$
D\left(\hat{\rho}_{k}, \rho_{k}\right)=\left\|\hat{\rho}_{k}-\rho_{k}\right\|_{F}^{2} /\left\|\rho_{k}\right\|_{F}^{2} .
$$

The online processing properties of the proposed QSE-OADM are campared with online proximal gradient-ADMM (OPG-ADMM) [22] and matrix exponential gradient (MEG) [21] in this paper. As we have mentioned in Section. 1, since Yang's CVX-LS algorithm [19] requires multiple iterations for each sampling time, which is time-consuming for online processing. Thus CVX-LS will not be compared in this paper. Similar to decomposing the objective function (11) into two subproblems about the density matrix $\hat{\rho}$ and measurement noise $\hat{e}$, OPG-ADMM uses the online proximal gradient descent method to approximately solve the $\hat{\rho}$-subproblem, which adds a proximal term to perform a proximal gradient update, and then satisfies the quantum state constraints by solving a semidefinite programming problem. The objective function of MEG consists of two terms, the first term is the Umegaki's quantum relative entropy between the estimated $\hat{\rho}$ and the previous estimate $\hat{\rho}_{k-1}$, and the second term is the square of the measurement error of the estimated state. Without projection operations, MEG guarantees that the estimated state meets the quantum state constraints through the exponential and trace normalization of the density matrix. However, the exponential of density matrix involves singular value decomposition, whose computational complexity is consistent with the projection operations in $\hat{\rho}$-subproblem.

There are three parameters involved in QSE-OADM: the weight parameters $w$ and $\gamma$; the penalty parameter $\alpha$ of OADM. In the experiments, they can be choosed as: $w=0.1$ and $\gamma=\sqrt{d} / k$; the penalty parameter $\alpha=2$. The parameters of OPG-ADMM and MEG are hand-tuned to the best. All simulations are conducted in MATLAB R2016a, running in Inter Core i7-8750M CPU, clocked at 2.2GHz, with a memory of 16GB.

\subsection{Impact of the estimated initial state on QSE-OADM, OPG-ADMM, and MEG}

For the state real-time tracking algorithm, different initial states of the estimation affect the algorithm performance. When the initial state deviates from the actual initial state greatly, it may lead to the accumulation of estimation errors and failure of accurate state estimation timely. Moreover, in order to intuitively reflect the estimation results, we choose the 1-qubit system, whose evolution trajectory can be clearly drawn on a Bloch sphere. Thus, we compare the proposed QSE-OADM with OPG-ADMM and MEG algorithms in two different estimated initial states $[0.75, \sqrt{3} / 4 ; \sqrt{3} / 4,0.25]$ and $[0.5,(1+i) /(\sqrt{8}) ;(1-i) /(\sqrt{8}), 0.5]$ (Relative to the true initial state $\rho_{1}$, one is far and the other is close.). The size of the sliding window is selected as $l=16$, which is shown to be sufficient to reconstruct the density matrix of 1-qubit system.

Fig. 1 depicts the trajectories of evolving quantum states and state estimates, in which the true quantum states are time-varying and evolve freely from the surface to the center of the Bloch sphere, whose evolution trajectory is represented by the red solid line. Correspondingly, the trajectory of time-varying estimated states by online algorithms is represented by the blue dashed line. For the number of samplings that the 
fidelity of the estimated state continuously greater than $99 \%$ in different estimated initial states, QSE-OADM is 3 (the fidelity is $99.71 \%$ ) and $3(99.48 \%)$; OPG-ADMM is $11(99.12 \%)$ and $13(99.63 \%)$; and MEG is $10(99.67 \%)$ and $14(99.87 \%)$, respectively. Compared with OPG-ADMM and MEG, the proposed QSEOADM can realize real-time state estimation accurately after just 3 steps for different estimated initial states. Thus, QSE-OADM is more robust to the estimated initial state.

\subsection{Efficiency properties of QSE-OADM, OPG-ADMM, and MEG}

In order to verify the efficiency of the online QSE algorithms, we compare the online estimation performance of QSE-OADM, OPG-ADMM, and MEG algorithm with different sliding window sizes. The size of the sliding window can be considered as a tradeoff between estimation accuracy and computational effort. At each window size $l$, the comparison criterion is the minimum number of sampling times $k_{\min }$ required for the normalized distance $D\left(\hat{\rho}_{k}, \rho_{k}\right)$ to be less than the baseline 0.1 . It is worth noting that $k_{\text {min }}$ is the comprehensive embodiment of the accuracy and tracking speed of the online state estimation algorithm. The number of $k_{\min }$ is expected to be smaller, indicating that the dynamic state can be reconstructed efficiently. In this experiment, for a 4-qubit system, the size of the sliding window is taken as $l=1, \ldots, 100$. Fig. 2 depicts the average of $k_{\min }$ and standard deviation range for each window size of the three algorithms running 10 times, respectively.

From Fig. 2, our findings are classified into two cases:

1) With the increase of the sliding window size, the number of sampling times $k_{\min }$ gradually decreases and tends to be stable. As shown by the black horizontal dotted lines in Fig. 2, the stable sampling times $k_{\text {min }}$ of QSE-OADM, OPG-ADMM, and MEG are 71, 170, and 182 respectively, which shows that QSE-OADM can realize high-precision online quantum state estimation with the lowest sampling steps.

2) We can also find that the minimum sliding window sizes of the QSE-OADM, OPG-ADMM, and MEG to achieve stable sampling times is 68, 80, and 85, respectively, from which one can see that QSE-OADM has the lowest number of samples, which indicates that compared with OPG-ADMM and MEG, QSE-OADM has more effective utilization of the measurement information.

\subsection{Online processing properties of QSE-OADM, OPG-ADMM, and MEG}

To verify the online processing performance, under the same sliding window size $l=70$, we compare the proposed QSE-OADM with OPG-ADMM and MEG algorithms in the online QSE of a 4-qubit system. Fig. 3 depicts the normalized distance $D\left(\hat{\rho}_{k}, \rho_{k}\right)$ with respect to the number of samplings in the online estimation process.

We compare the normalized distances of QSE-OADM, OPG-ADMM, and MEG algorithms at the 100th online estimation, which are 0.0541, 0.1589, and 0.2118, respectively, from which one can see that the normalized estimation error of QSE-OADM is less than 3 to 4 times that of OPG-ADMM and MEG in the same sampling times. Furthermore, the corresponding fidelities of QSE-OADM, OPG-ADMM, and MEG algorithms are $98.77 \%, 83.24 \%$, and $76.39 \%$, respectively. Thus, under the same sampling times, the estimation accuracy of QSE-OADM is the highest among three algorithms.

The average running time of QSE-OADM, OPG-ADMM, and MEG is $(4.19 \pm 0.41) \times 10^{-4} s,(9.75 \pm$ $0.12) \times 10^{-4} s$, and $(1.21 \pm 0.06) \times 10^{-3} s$, respectively. At the same time, from Fig. 3 QSE-OADM, OPGADMM, and MEG algorithms need 71, 161 and 191 sampling times respectively to reach the normalized distance less than 0.1 (or the fidelity is more than 97.57\%). One can see that QSE-OADM achieves very high precision online state tracking with the least average running time and the lowest sampling times. Therefore, the merit of the proposed algorithm is that it can track the dynamic quantum state fast and efficiently, which embodies its superiority in online QSE. Because the proposed algorithm in this paper can be online realized, which may be applied in the practical quantum system experiments, especially in the quantum state feedback control systems for high accurate manipulation of quantum states. 


\section{Conclusion}

In this paper, a novel online algorithm QSE-OADM in a noisy CWM process was developed, which processed the measurement values in a serialized manner, and exactly solved two sub-problems in every estimation. Furthermore, for achieving high estimation accuracy and improving efficiency, the sliding window of measurements was adopted. The algorithm we proposed was efficient and fast to estimate the dynamic quantum state. Numerous experiments are supportive of the potential merits of the method as an online solution for state estimation in multi-qubit quantum systems.

\section{Acknowledgment}

This work was supported by the National Natural Science Foundation of China under Grants No. 61973290 and 61720106009.

\section{References}

[1] M. Paris, J. Rehacek, Quantum state estimation 649 (2004) 59-112.

[2] Y. Eldar, A. Oppenheim, Quantum signal processing, IEEE Signal Processing Magazine 19 (2002) $12-32$.

[3] A. C. Doherty, K. Jacobs, Feedback control of quantum systems using continuous state estimation, Physical Review A 60 (1999) 2700.

[4] A. Kalev, I. Hen, Fidelity-optimized quantum state estimation, New Journal of Physics 17 (2015) 093008.

[5] D. Gross, Y.-K. Liu, S. T. Flammia, S. Becker, J. Eisert, Quantum state tomography via compressed sensing, Physical Review Letters 105 (2010) 150401.

[6] M. Cramer, M. B. Plenio, S. T. Flammia, R. Somma, D. Gross, S. D. Bartlett, O. Landon-Cardinal, D. Poulin, Y.-K. Liu, Efficient quantum state tomography, Nature Communications 1 (2010) 1-7.

[7] A. Silberfarb, P. S. Jessen, I. H. Deutsch, Quantum state reconstruction via continuous measurement, Physical Review Letters 95 (2005) 030402.

[8] G. A. Smith, A. Silberfarb, I. H. Deutsch, P. S. Jessen, Efficient quantum-state estimation by continuous weak measurement and dynamical control, Physical Review Letters 97 (2006) 180403.

[9] A. Korotkov, D. Averin, Continuous weak measurement of quantum coherent oscillations, Physical Review B 64 (2001) 165310.

[10] J. F. Ralph, K. Jacobs, C. D. Hill, Frequency tracking and parameter estimation for robust quantum state estimation, Physical Review A 84 (2011) 052119.

[11] S. Boyd, N. Parikh, E. Chu, B. Peleato, J. Eckstein, et al., Distributed optimization and statistical learning via the alternating direction method of multipliers, Foundations and Trends $\AA$ in Machine learning 3 (2011) 1-122. 
[12] K. Li, S. Cong, A robust compressive quantum state tomography algorithm using admm, IFAC Proceedings Volumes 47 (2014) 6878-6883.

[13] K. Zheng, K. Li, S. Cong, A reconstruction algorithm for compressive quantum tomography using various measurement sets, Scientic Reports 6 (2016) 38497.

[14] J. Zhang, K. Li, S. Cong, H. Wang, Efficient reconstruction of density matrices for high dimensional quantum state tomography, Signal Processing 139 (2017) 136-142.

[15] J. Zhang, K. Li, S. Cong, Fast algorithm of high-dimensional quantum state estimation via low measurement rates, 2017 11th Asian Control Conference (ASCC) (2017) 2558-2562.

[16] J. Zhang, S. Cong, Q. Ling, K. Li, An efficient and fast quantum state estimator with sparse disturbance, IEEE Transactions on Cybernetics 49 (2018) 2546-2555.

[17] J. Zhang, S. Cong, Q. Ling, K. Li, H. Rabitz, Quantum state filter with disturbance and noise, IEEE Transactions on Automatic Control 65 (2020) 2856-2866.

[18] Z. Leghtas, M. Mirrahimi, P. Rouchon, Back and forth nudging for quantum state estimation by continuous weak measurement, Proceedings of the 2011 American Control Conference (2011) 43344339.

[19] J. Yang, S. Cong, S. Kuang, Real-time quantum state estimation based on continuous weak measurement and compressed sensing, Proceedings of the International MultiConference of Engineers and Computer Scientists (IMECS) 2 (2018) 499-504.

[20] M. Grant, S. Boyd, Y. Ye, Cvx: Matlab software for disciplined convex programming, 2008.

[21] A. Youssry, C. Ferrie, M. Tomamichel, Efficient online quantum state estimation using a matrixexponentiated gradient method, New Journal of Physics 21 (2019) 033006.

[22] K. Zhang, S. Cong, Y. Tang, N. M. Freris, An efficient online estimation algorithm for evolving quantum states, the 28th European Signal Processing Conference (2020) Amsterdam, Netherlands, to be published.

[23] H. Wang, A. Banerjee, Online alternating direction method, 29th International Conference on Machine Learning, ICML 2012 (2012) 1119-1126.

[24] S. Harraz, S. Cong, State transfer via on-line state estimation and lyapunov-based feedback control for a n-qubit system, Entropy 21 (2019) 751-762.

[25] S. Cong, Y. Tang, S. Harrz, K. Li, J. Yang, On-line quantum state estimation using continuous weak measurement and compressed sensing, Science China Information Sciences 64 (2021) 189202.

[26] A. J. Faria, H. M. França, C. P. Malta, R. C. Sponchiado, Critical assessment of the schrödinger picture of quantum mechanics, Physical Review A 305 (2002) 322-328.

[27] J. Bosse, O. Rabaste, Subspace rejection for matching pursuit in presence of unresolved targets, IEEE Transactions on Signal Processing 66 (2018) 1997-2010.

[28] F. Ding, Decomposition based fast least squares algorithm for output error systems, Signal Processing 93 (2013) 1235-1242.

[29] Boyd, Vandenberghe, Faybusovich, Convex optimization, IEEE Transactions on Automatic Control 51 (2006) 1859-1859. 

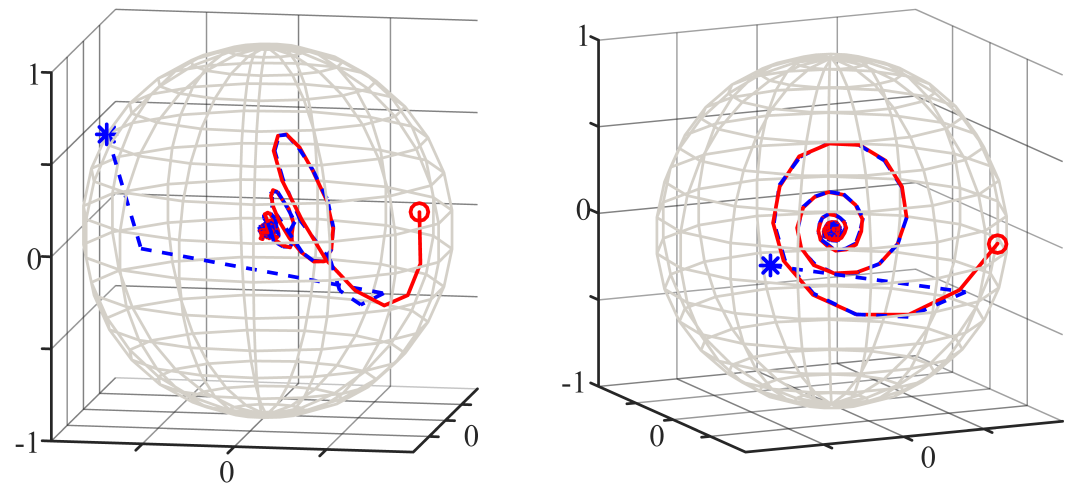

(a) QSE-OADM
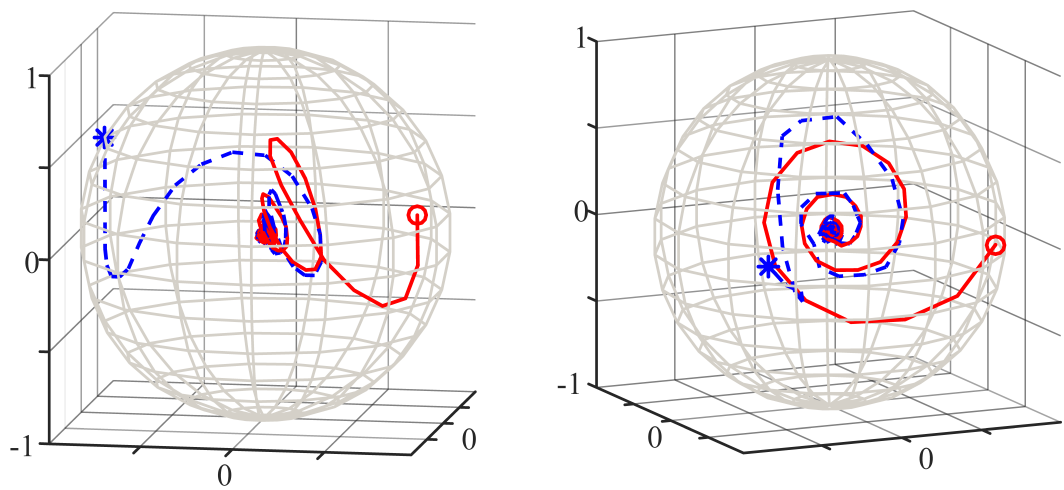

(b) OPG-ADMM
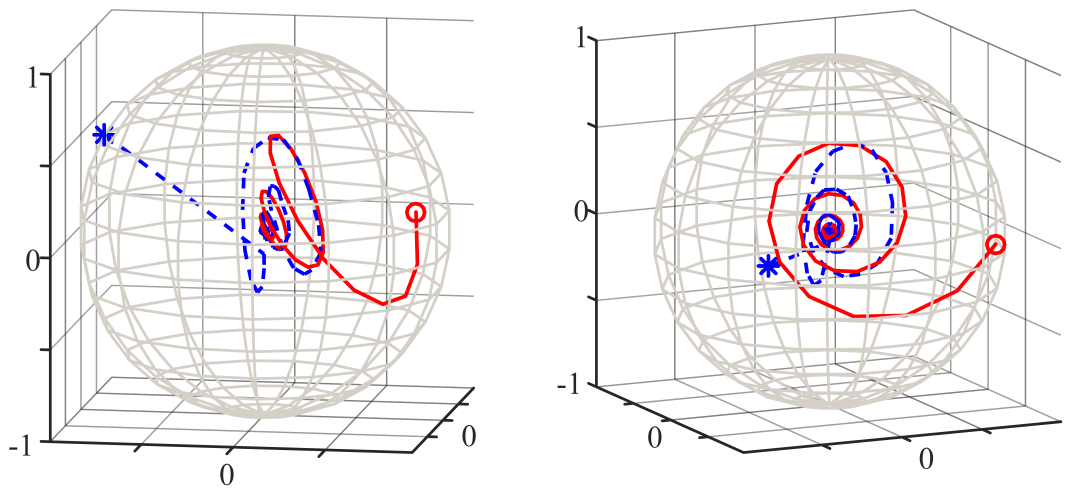

(c) MEG

Fig. 1: Trajectories of evolving quantum states and state estimates. Online state estimation trajectories of the three algorithms under two different estimated initial states. The red circle and the blue star represent the initial values of true and estimated states, respectively. The red solid line and the blue dashed line in the Bloch sphere are the true quantum state trajectory and the estimated state trajectory, respectively. 


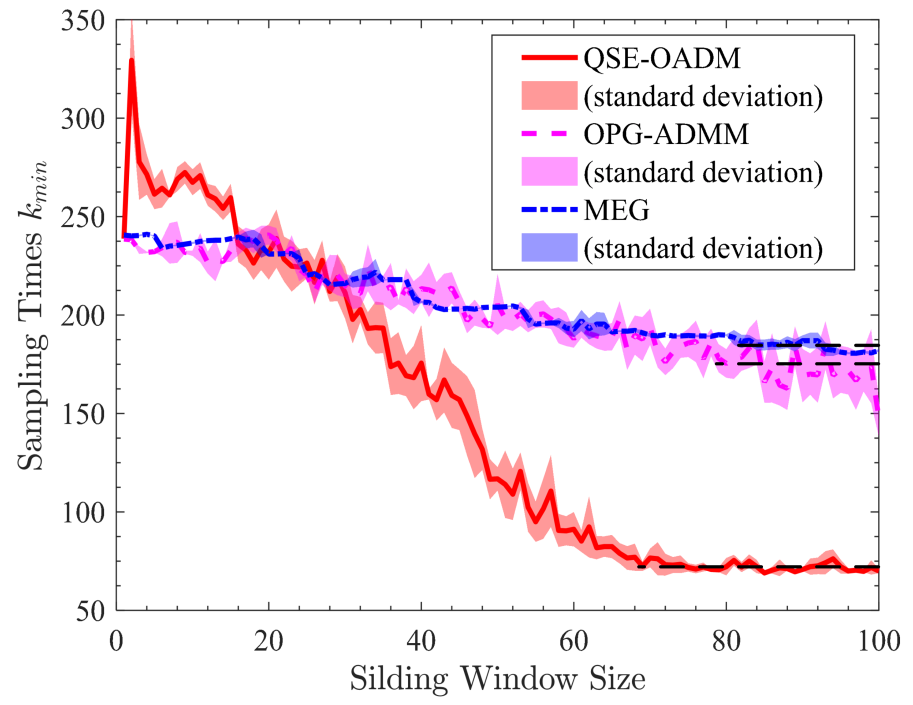

Fig. 2: Comparison of online estimation efficiency of QSE-OADM, OPG-ADMM, and MEG that run for the sliding window size $l=1, \ldots, 100 . k_{\min }$ indicates the minimum number of sampling times required to satisfy $D\left(\hat{\rho}_{k}, \rho_{k}\right)<0.1$ for each window size. 


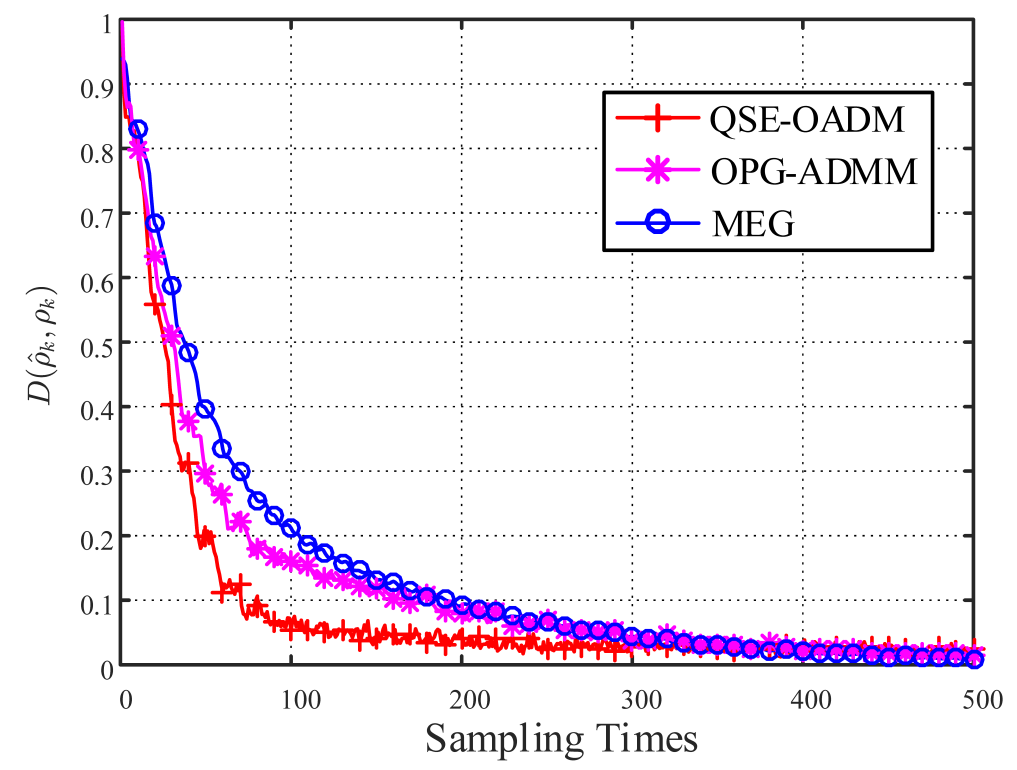

Fig. 3: Comparisons of QSE-OADM, OPG-ADMM, and MEG algorithms in online quantum state reconstruction performances that run for the sliding window size $l=70$. 